Please do not remove this page

RMIT

UNIVERSITY

\title{
Photophysics of chromium-related diamond single-photon emitters
}

Aharonovich, I; Castelletto, Stefania; Simpson, David; Greentree, Andrew; Prawer, Steven

https://researchrepository.rmit.edu.au/esploro/outputs/9921858160701341/filesAndLinks?institution=61RMIT_INST\&index=null

Aharonovich, I., Castelletto, S., Simpson, D., Greentree, A., \& Prawer, S. (2010). Photophysics of chromium-related diamond single-photon emitters. Physical Review A, 81(4), 1-7.

https://doi.org/10.1103/PhysRevA.81.043813

Published Version: https://doi.org/10.1103/PhysRevA.81.043813

Repository homepage: https://researchrepository.rmit.edu.au

(c) 2010 The American Physical Society

Downloaded On 2023/04/26 20:02:45 +1000

Please do not remove this page 
Thank you for downloading this document from the RMIT Research Repository.

The RMIT Research Repository is an open access database showcasing the research outputs of RMIT University researchers.

RMIT Research Repository: http://researchbank.rmit.edu.au/

\section{Citation:}

Aharonovich, I, Castelletto, S, Simpson, D, Greentree, A and Prawer, S 2010, 'Photophysics of chromium-related diamond single-photon emitters', Physical Review A, vol. 81, no. 4, 043813, pp. 1-7.

See this record in the RMIT Research Repository at:

http://researchbank.rmit.edu.au/view/rmit:15475

Version: Published Version

Copyright Statement: ㄷ 2010 The American Physical Society

Link to Published Version:

http://dx.doi.org/10.1103/PhysRevA.81.043813 


\title{
Photophysics of chromium-related diamond single-photon emitters
}

\author{
I. Aharonovich, ${ }^{*}$ S. Castelletto, D. A. Simpson, A. D. Greentree, and S. Prawer \\ School of Physics, The University of Melbourne, 3010 Victoria, Australia
}

(Received 20 December 2009; published 13 April 2010)

\begin{abstract}
A detailed study of the photophysical properties of several chromium-related color centers produced within chemical vapor deposition diamond is presented. These emitters show narrow luminescence lines in the range of $740-770 \mathrm{~nm}$. Single-photon emission was verified with continuous and pulsed excitation with detected emission rates at saturation in the range of $(2-3) \times 10^{6}$ counts/s, while direct lifetime measurements reveal excited state lifetimes for the distinct centers ranging 1-14 ns. In addition, a number of quantum emitters demonstrate two-level behavior with no bunching present in the second-order correlation function. The three-level systems revealed typically photoluminescence lines with width half-maximum of $\sim 4 \mathrm{~nm}$ while the two-level emitters have full width half-maximum of $\sim 10 \mathrm{~nm}$ at room temperature. In addition, the quantum efficiency of the two-level system was measured to be four times higher than that of the three-level system.
\end{abstract}

DOI: 10.1103/PhysRevA.81.043813

\section{INTRODUCTION}

The emerging field of quantum optics has established a demand for an accessible solid-state system which can generate a stream of single photons on demand [1]. Although single-photon emission has been demonstrated from quantum dots (QD) [1,2], single molecules [3], and nanowires [4], the operation of those systems is often limited by temperature. Diamond crystals, on the other hand, offer a promising platform for the generation of robust, photostable, single photons at room temperature [5,6]. Nevertheless, out of more than 500 existing optical centers in diamond [7], only three centers with a known atomic structure have demonstrated single-photon emission, namely, the nitrogen-vacancy $(\mathrm{N}$ $V)$ complex [6,8], the silicon-vacancy ( $\mathrm{Si}-V)$ complex [9], and the nickel-nitrogen complex (NE8), which contains four equivalent nitrogen atoms [10-12].

Nickel-related optically active defects were fabricated by incorporation of $\mathrm{Ni}$ during the chemical vapor deposition (CVD) growth $[13,14]$ or by a direct implantation of Ni into the grown crystals [15]. Increased studies in the formation of diamond color centers unveiled single-photon sources emitting $\sim 10^{6}$ photons/s [16,17]. These new centers are comparable with QD in terms of brightness [1], but have the tremendous advantage of stable operation at room temperature. This breakthrough has opened a new avenue to investigate essentially an unknown group of single-photon emitters in diamond.

The two-level nature of selected single-photon emitters at different wavelengths could be a significant system for metrological applications, because the autocorrelation function does not exhibit bunching even at saturation. One can envisage that single-photon sources possessing two-level behavior could be implemented as a "single-photon standard," able to link classical radiometric measurements to fundamental quantum optical entities. In particular, in the long term such a singlephoton standard could contribute to a redefinition of the standard units for optical radiation in terms of the "quantum candela" [18]. The controlled fabrication of the $\mathrm{MHz}$ class of diamond single-photon emitters, together with a recent emission enhancement by coupling the light to plasmonic

\footnotetext{
*i.aharonovich@pgrad.unimelb.edu.au,sacas@unimelb.edu.au
}

PACS number(s): 42.50.Ar, 78.67.Bf, 78.47.jd, 81.05.ug

structures [19] or cavities [20,21], is expected to progress the technology beyond the point in which they can become practical quantum information and metrology devices [22]. In particular, the high count rates and the variety of emission lines associated with the presented sources could contribute to fill the gap between conventional radiometry and quantum radiometry as recently reported [23].

Here we present a detailed and comprehensive study of the photophysical properties of the family of $\mathrm{Cr}$-related singlephoton emitters reported in [17]. The present manuscript reveals the difference in the photophysical properties of a closely related family of $\mathrm{Cr}$ centers. Surprisingly, although the photoluminescence (PL) emission is concentrated within a range of $30 \mathrm{~nm}$, the properties of the emitters such as lifetime, brightness, photon statistics (two or three level) and quantum efficiency are varying from center to center. Identifying such a variation of optical properties in one family of $\mathrm{Cr}$ centers is unique and its classification is essential for further implementation of these emitters in practical devices. We concentrate on the typical representative PL lines in the range of 740-770 nm, which exhibit single-photon emission with count rates ranging from (1.3-3.2) $\times 10^{6}$ counts $/ \mathrm{s}$ at saturation. In extending our previous work [17], in this paper we have gathered an extended statistics on the photophysical properties of various emitters as well as measured the metastable state lifetime (for the three-level emitters). This paper is organized as follows: Sec. II will discuss the theoretical background, Sec. III will describe the experiment, and in Sec. IV we will discuss the results.

\section{THEORETICAL BACKGROUND}

The physics of the PL emitted from a single diamond color center can be described within the framework either of a twolevel system comprising a ground and excited state, and in many instances as a three-level system $[5,11,24]$, whereby the single center is excited from its ground state to the excited state, with a third longer-lived state providing an additional decay path from the excited state, as seen in Fig. 1.

The rate equations describing the populations of the twoand three-level systems can be written in matrix form as

$$
\left(\begin{array}{l}
\dot{n}_{1} \\
\dot{n}_{2}
\end{array}\right)=\left(\begin{array}{cc}
-r_{12} & r_{12} \\
r_{12} & -r_{21}
\end{array}\right)\left(\begin{array}{l}
n_{1} \\
n_{2}
\end{array}\right)
$$



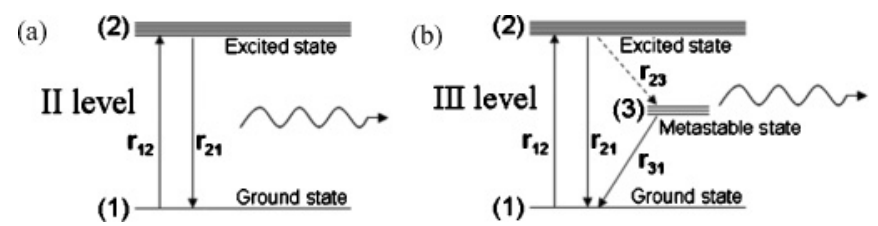

FIG. 1. Schematic diagram of a (a) two-level and (b) three-level system, where $r_{i j} i, j=1,2,3$ are the transition rates from level $(i)$ to level $(j)$.

and

$$
\left(\begin{array}{c}
\dot{n}_{1} \\
\dot{n}_{2} \\
\dot{n}_{3}
\end{array}\right)=\left(\begin{array}{ccc}
-r_{12} & r_{21} & r_{31} \\
r_{12} & -r_{21}-r_{23} & 0 \\
0 & r_{23} & -r_{31}
\end{array}\right)\left(\begin{array}{l}
n_{1} \\
n_{2} \\
n_{3}
\end{array}\right) .
$$

$r_{i j}$ with $i, j=1,2,3$ are the transition rates from level $(i)$ to level $(j)$, and $n_{i}(t)$ is the population in the $i$ th level. By solving Eqs. (1) and (2) with the initial condition $n_{1}=1, n_{2}=$ $0, n_{3}=0$ (i.e., the system is prepared in the ground state), the instantaneous emission probability of a photon being emitted from the excited state, $n_{2}(t)$, can be obtained. The analytical expression of the second-order correlation function $g^{(2)}(\tau)=\left\langle n_{2}(\tau)\right\rangle /\left\langle n_{2}(\infty)\right\rangle$ for the two- and three-level [11,24] cases are then, respectively,

$$
g^{(2)}(\tau)=1-\exp \left(-\lambda_{1} \tau\right)
$$

where $\lambda_{1}=r_{12}+r_{21}$ and

$$
g^{(2)}(\tau)=1-(1+a) \exp \left(-\lambda_{1} \tau\right)+a \exp \left(-\lambda_{2} \tau\right),
$$

where $\lambda_{2}=r_{31}+r_{23} r_{12} / \lambda_{1}$, and $a=r_{12} r_{23} /\left(\lambda_{1} r_{31}\right)$.

The decay rate $\lambda_{1}$ depends on the excitation optical power, $P_{\mathrm{opt}}$, and it can be rewritten as

$$
\lambda_{1}=r_{21}^{0}\left(1+\alpha P_{\mathrm{opt}}\right)
$$

where $r_{21}^{0}$ is the inverse of the excited state lifetime $\left(\tau_{\mathrm{rad}}\right)$ and $\alpha$ is a fitting parameter, accounting for the optical excitation power dependence of the excited state decay rate.

Similarly, for the three-level system, the decay rate $\lambda_{2}$, assuming $r_{23}$ constant with the excitation power, can be written in terms of $r_{31}^{0}$ in the limit of zero optical power [11] as

$$
\lambda_{2}=r_{31}^{0}\left(1+\beta P_{\mathrm{opt}}\right)+\frac{r_{23} r_{12}}{r_{21}^{0}\left(1+\alpha P_{\mathrm{opt}}\right)},
$$

where $\beta$ is a fitting parameter that takes into account the variation of the decay rate from the metastable state to the ground state with the optical excitation power. This parameter is relevant, since it justifies a power dependence effect in deshelving the metastable level; this dependence has also been observed for single molecules [25].

With an accurate determination of the second-order correlation function one can determine the individual decay rates involved in the system and gain an understanding of the energy level structure. Upon inspection of the two- and three-level autocorrelation functions, there is a clear distinction in the shape and nature of the exponential component. In the two-level case, the function exhibits a simple exponential behavior at a rate equivalent to the fluorescence decay rate of the excited state in the limit of zero optical excitation (i.e., $r_{12} \rightarrow 0$ ), with the exponential asymptotic to a $g^{(2)}(\tau)$ value of 1 . The three-level expression, on the other hand, contains two exponential components each with a characteristic time constant. Depending on the transition rates from the excited state to the shelving state and shelving state back to the ground state, $g^{(2)}(\tau)$ can increase beyond 1 for times $>\tau_{\text {rad }}$, before going to the asymptote of 1 at times $\gg \tau_{\text {rad }}$.

The phenomenon of $g^{(2)}(\tau)>1$ is commonly termed "photon bunching" and this behavior enables a clear distinction to be made between two- and three-level systems. The second-order correlation function describes the probability of detecting a photon with a delay time $\tau$ after one photon has been detected at $\tau=0$. Hence, the bunching effect describes an enhanced probability to detect a photon at short times than at longer times. Indeed, after the system undergoes a transition to its shelving state, which has a longer lifetime, there is a longer interval between the photons. Once the system relaxes to the ground state and undergoes a full emission cycle again, the normal photon rate is achieved again. The waiting interval while the system is in the metastable state thus creates the bunching effect of the $g^{(2)}(\tau)$.

The measurement of $g^{(2)}(\tau)$ is performed typically using a Hanbury-Brown and Twiss (HBT) interferometer whereby the coincidence events of two detectors are measured. In practice, the temporal jitter of the detectors and electronics can be ignored provided that the lifetime is much longer than the temporal jitter; with the identification of emitting centers with increasing short fluorescence lifetimes [9,11,13], the effect of the temporal jitter on the measured autocorrelation function $g_{\text {meas }}^{(2)}(\tau)$ must be taken into account. Therefore, the measured autocorrelation function is given by the convolution of Eqs. (3) and (4) with the instrument time response function $J(\tau)$ :

$$
g_{\text {meas }}^{(2)}(\tau)=\int_{-\infty}^{\infty} d \tau^{\prime} g^{(2)}\left(\tau^{\prime}\right) J\left(\tau-\tau^{\prime}\right)
$$

The majority of single-photon emitters identified in diamond (such as Si-V [9], N-V [6], and the NE8 [10,11]) have been attributed to three-level systems which suffer from quenching due to the presence of a shelving state. However, the recent identification of highly efficient two-level emitters in diamond $[16,17]$ can have a significant role in determining more accurately the fluorescence quantum yield. The fluorescence quantum yield, $\eta_{\mathrm{QE}}$, of an emitter is defined as the probability of an absorbed pump photon resulting in an emitted photon, and can be written as

$$
\eta_{\mathrm{QE}}=\frac{k_{\mathrm{rad}}}{k_{\mathrm{rad}}+k_{\mathrm{nrad}}+k_{\mathrm{ISC}}},
$$

where $k_{\text {rad }}$ and $k_{\text {nrad }}$, are the radiative and nonradiative decay rates, respectively, and $k_{\text {ISC }}$ is the intersystem crossing decay rate. For a two-level emitter $k_{\mathrm{ISC}}=0$.

We can deduce the number of emitted photons or the fluorescence count rate of a two-level system by solving the rate equations in Eq. (1):

$$
\phi=\frac{\phi_{\infty} P_{\mathrm{opt}}}{P_{\mathrm{sat}}+P_{\mathrm{opt}}},
$$

where $\phi$ represents the single-photon count rate. $\phi_{\infty}=$ $r_{21}^{0} \eta \eta_{\mathrm{QE}}$ is the saturation count rate for $P_{\mathrm{opt}} \rightarrow \infty$, where $\eta$ represents the total collection efficiency and $P_{\text {sat }}$ is the optical saturation power. 

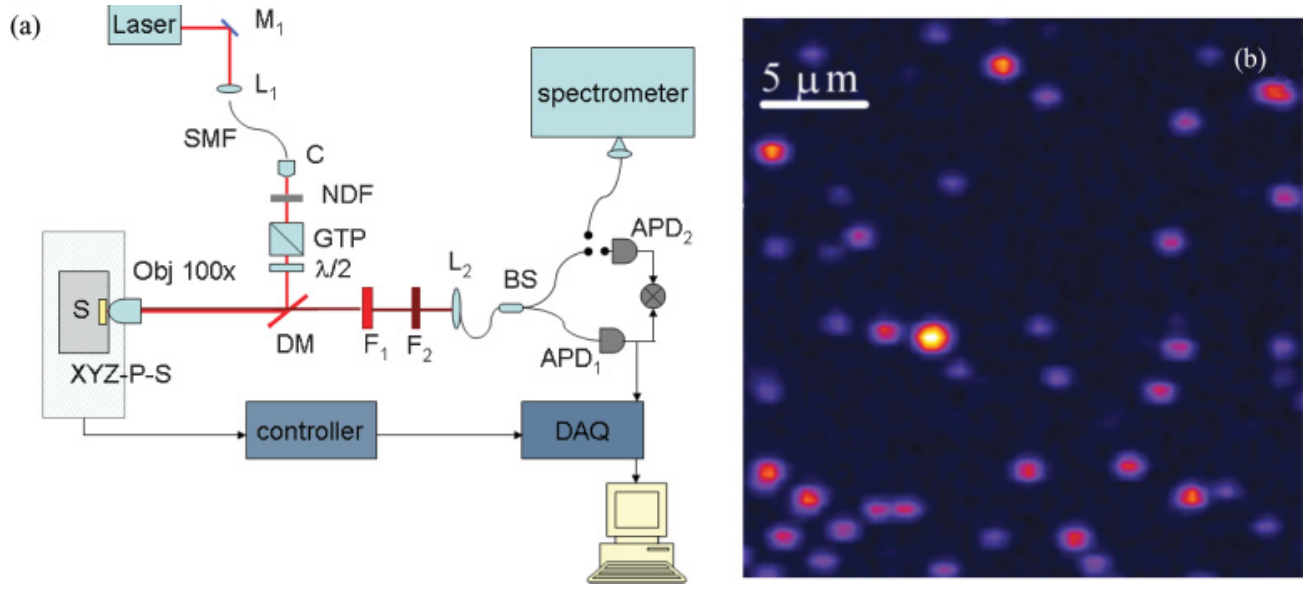

FIG. 2. (Color online) (a) Experimental setup. A cw diode laser at $682 \mathrm{~nm}$ and a pulsed 690-nm laser were alternatively coupled to a single-mode fiber, and then collimated (C) and polarized by a Glan-Taylor polarizer (GTP). The laser polarization was varied by a half-wave plate $(\lambda / 2)$. A variable neutral density filter (NDF) was used to change the excitation power. Samples (S) were excited by focusing the laser light by a high-numerical-aperture (0.9) objective $(\mathrm{Obj})$ with $100 \times$ magnification. A dichroic mirror (DM) transmitting from $720 \mathrm{~nm}$, was used to separate the laser line from the sample fluorescence emission, when collected back from the same objective. $F_{1,2}$ are band-pass filters, $794 \pm 80 \mathrm{~nm}$ and alternatively $760 \pm 12 \mathrm{~nm}$ or $740 \pm 12 \mathrm{~nm}$, to isolate the single-photon emission lines. A 100-mm focal length lens was used to send the single-photon emission to a multimode fiber, providing an aperture for the confocal imaging. Finally a 50:50 fiber beam splitter was used to verify the single-photon emission by performing the autocorrelation between two low-dark counts (150 counts/s) single-photon counting modules (APD). The samples were mounted on a Physics Instruments $X Y Z$ piezo stage in closed loop operation. (b) Confocal image of $20-\times 20-\mu \mathrm{m}^{2}$ showing bright spots which correspond to color centers within a diamond nanocrystal.

In the three-level case, the fluorescence count rate can be expressed in terms of the fluorescence quantum yield by solving the rate equations in Eq. (2):

$$
\phi=\eta_{\mathrm{QE}} \eta \frac{r_{21}}{1+\frac{r_{21}}{r_{12}}+\frac{r_{23}}{r_{31}}} .
$$

From inspection of Eq. (10) it is clear that at a constant excitation the effect of photon bunching becomes stronger with increasing $\frac{r_{23}}{r_{31}}$, while the source efficiency decreases.

\section{EXPERIMENT}

The CVD diamond nanocrystals employed in this work were grown to an average size of few hundreds of nanometers from diamond seeds $(4-6 \mathrm{~nm})$ on a sapphire substrate using a microwave plasma-enhanced CVD technique $(900 \mathrm{~W}$, 150 Torr) [26]. The Cr-related single-photon emitters are fabricated through a diffusion of Cr into the crystals [13] from the sapphire substrate during the growth. The concentration of $\mathrm{Cr}$ atoms in the underlying substrate is at the ppm level, and growth on substrates which do not contain $\mathrm{Cr}$ (such as silica) did not produce the centers reported here. A home-built confocal microscope with a spatial resolution $\sim 400 \mathrm{~nm}$ and HBT interferometer were used to identify the emitting centers and measure the time correlation of PL intensity [Fig. 2(a)]. A fiber-coupled cw diode laser emitting at $682 \mathrm{~nm}$ and a $690-\mathrm{nm}$ pulsed diode with 200-ps pulse width (repetition rate from $10 \mathrm{MHz}$ up to $80 \mathrm{MHz}$ ) were interchanged for excitation. The laser polarization was controlled by a Glan-Taylor polarizer and half-wave plate. The diamond sample was mounted on a piezo $X Y Z$ stage with $0.2-\mathrm{nm}$ resolution, allowing $100-\times$ $100-\mu \mathrm{m}^{2}$ scans. The unwanted residual laser line was eliminated by a dichroic beam splitter and an $F_{1}$ broad $(10 \mathrm{~nm}$
FWHM) band-pass filter centered at 740 or $760 \mathrm{~nm}$ depending on the PL from a specific crystal. The PL from the emitting centers was then coupled into a $62.5-\mu \mathrm{m}$ core multimode fiber, which acts as an aperture. A 50:50 fiber-coupled beam splitter guided the photons to two single-photon counting detectors (APDs) and their outputs were sent to the start and stop inputs of the time correlator card. The PL was recorded without the bandpass $F_{1}$ filter using a fiber-coupled spectrometer with a cooled CCD array. All measurements were performed at room temperature.

\section{RESULTS}

More than 10 different sapphire substrates were scanned, with crystals grown to a size of a few hundred nanometers. Figure 2(b) shows a typical confocal map of the diamond nanocrystals obtained by 682-nm laser excitation. The PL spectra of the bright spots revealed emission with zero phonon lines (ZPLs) centered at $744 \pm 2 \mathrm{~nm}(\mathrm{FWHM} \sim 11 \mathrm{~nm}), 749 \pm$ $2 \mathrm{~nm}(\mathrm{FWHM} \sim 4 \mathrm{~nm}), 756 \pm 2 \mathrm{~nm}(\mathrm{FWHM} \sim 11 \mathrm{~nm})$, and $764 \pm 2 \mathrm{~nm}(\mathrm{FWHM} \sim 10 \mathrm{~nm})$, as shown in Fig. 3. In some cases two or more of these lines were found in one crystal, as shown in the inset of Fig. 3. Note that the similarity in the emission wavelength around $770 \mathrm{~nm}$ between the reported Cr-related centers and the previously reported Ni-Si centers [15] is completely coincidental. No Ni implantation was employed during the fabrication of the $\mathrm{Cr}$ centers.

Antibunching measurements on crystals containing multiple emission lines generally showed multiple emitters with autocorrelation functions at the zero delay time of $g^{(2)}(0)>0.5$, with the intensity of the lines varying from crystal to crystal. These characteristic emission lines are in agreement with cathodo-luminescence lines from chromium-related centers in 


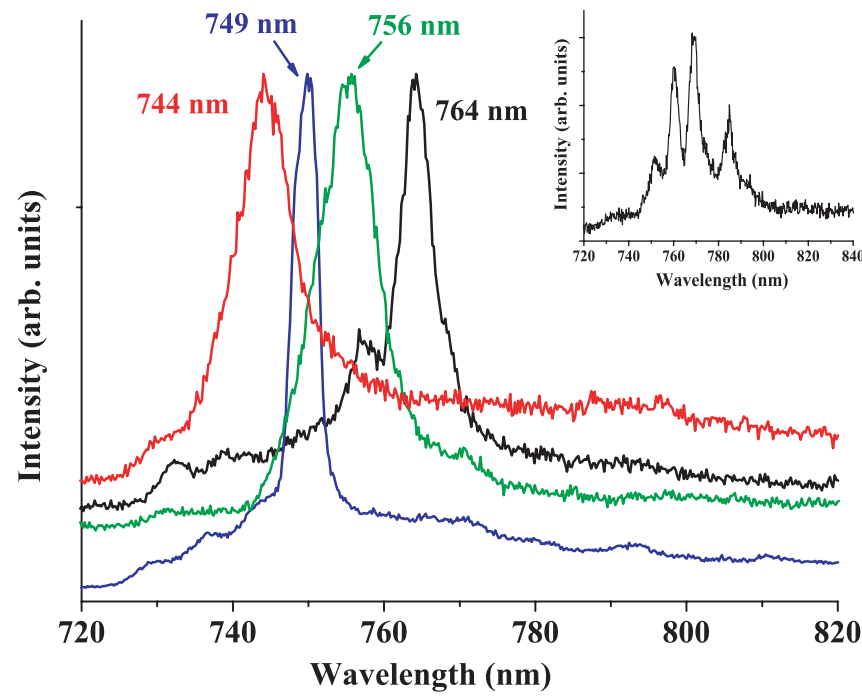

FIG. 3. (Color online) Typical normalized PL spectra recorded from individual CVD diamond nanocrystals as shown in the raster scan of the sample in Fig. 1. The peak emission lines centered at $744 \mathrm{~nm}$ (red), $749 \mathrm{~nm}$ (blue), $756 \mathrm{~nm}$ (green), and $764 \mathrm{mn}$ (black). In some cases a few PL lines could be found in one nanocrystal (inset). diamond, exhibiting narrow lines in the region of 740-770 nm [27].

Figure 4(a)-4(d) show the antibunching behavior of the PL lines centered at 744, 749, 756, and $764 \mathrm{~nm}$ measured with the HBT interferometer for excitation powers below and above the optical saturation power $P_{\text {sat }}$. The dip at zero delay time indicates a single-photon emitter. The raw coincidence data were corrected for the background as described in Ref. [6].

Due to the observed bunching, the center with a ZPL at $749 \mathrm{~nm}$ was fit by Eqs. (4) and (7), which describes the threelevel model. The other three centers, which exhibit two-level behavior, were fit by Eqs. (3) and (7). The deviation from zero of the autocorrelation function is attributed to the jitter of the electronics and detectors and residual polarization-dependent background.

The $g_{\text {meas }}^{(2)}(0)$ of the 744-, 749-, 756-, and 764-nm centers were $0.44,0.16,0.2$, and 0.09 , respectively. The excited state radiative lifetime of the 744-, 749-, 756- and 764-nm emitters were $3.8 \pm 0.3,1.1 \pm 0.1,3.7 \pm 0.5$, and $13 \pm 1 \mathrm{~ns}$, respectively, and were obtained by extrapolating the decay rate $\lambda_{1}$ to zero optical power; refer to Eq. (5) (see Fig. 5). We note that the emitter at $764 \mathrm{~nm}$ here presented is a different emitter from the one reported in [17], which has
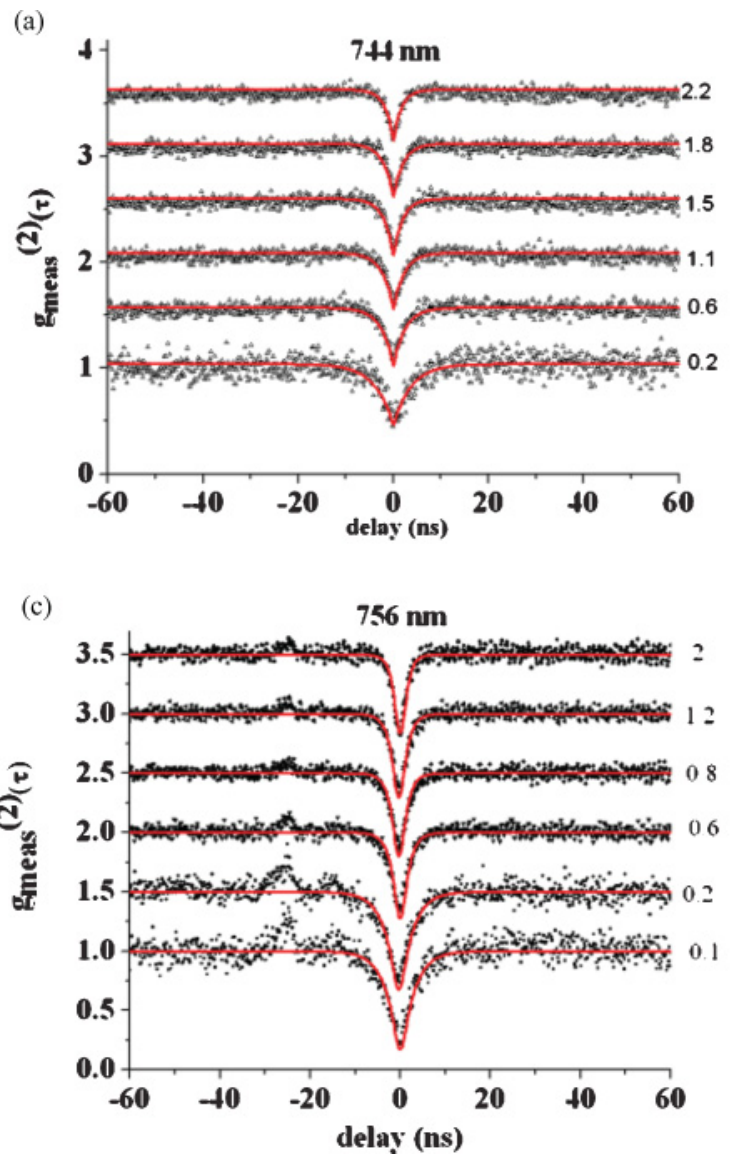

(b)

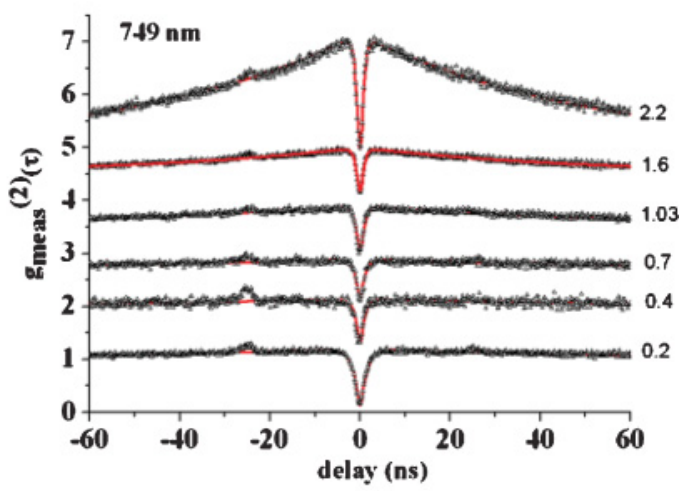

(d)

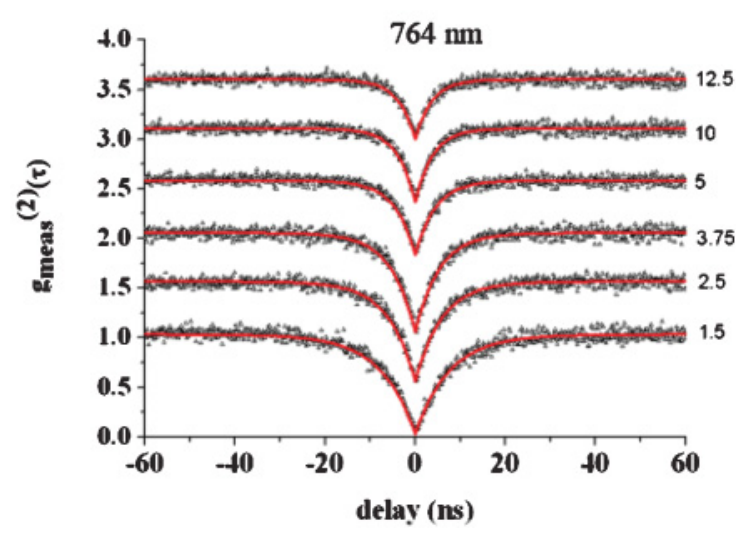

FIG. 4. (Color online) Background corrected second-order autocorrelation function $g_{\text {meas }}^{(2)}(\tau)$, measured with 154-ps coincidence time bin for $300 \mathrm{~s}$ at different optical powers for the (a) 744-nm line, (b) 749-nm line, (c) 756-nm line, and (d) 764-nm line. The data of the 749-nm line were fit by Eqs. (4) and (7), while the data of the 744-, 756-, and 764-nm lines were fit by Eqs. (3) and (7). The number to the right of the curves correspond to $P_{\text {opt }} / P_{\text {sat }}$. The peaks at $-20 \mathrm{~ns}$ and at $30 \mathrm{~ns}$ in Figs. 4(b) and 4(c) are due to crosstalk between the APDs, which has been removed by replacing a flat fiber patch cord with an angled one, eliminating the effect in the other figures. The plots in each graph were shifted for clarity. 


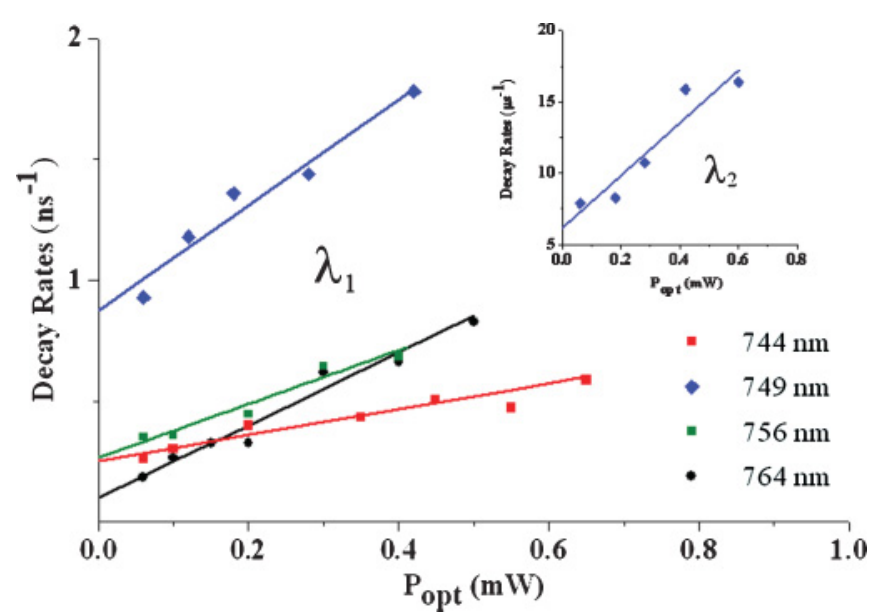

FIG. 5. (Color online) Estimated $\lambda_{1}$ parameters for the centers at $744 \mathrm{~nm}$ (red squares), $749 \mathrm{~nm}$ (blue diamonds), $756 \mathrm{~nm}$ (green triangles), and $764 \mathrm{~nm}$ (black circles) versus the optical power. At the limit of zero optical power the lifetimes of the centers are, respectively, $\left(r_{21}^{0}\right)^{-1}=3.8 \pm 0.3 \mathrm{~ns}, 1.1 \pm 0.1 \mathrm{~ns}, 3.7 \pm 0.5 \mathrm{~ns}$, and $13 \pm 1 \mathrm{~ns}$. The data were fit with Eq. (5). The behavior of $\lambda_{2}$ versus the optical power of the emitters at $749 \mathrm{~nm}$ is also shown in the inset and fit with Eq. (6); the estimated values $r_{31}^{0}=6.2 \mathrm{MHz}(161 \mathrm{~ns})$, and $r_{23}=0.89 \mathrm{MHz}(1.1 \mu \mathrm{s}), \alpha=2.5 \mathrm{~mW}^{-1}, \beta=3.1 \mathrm{~mW}^{-1}$.

instead a shorter lifetime and a narrower FWHM bandwidth $(\sim 5 \mathrm{~nm})$.

The fit applied to the second-order correlation function for the 749-nm emitter contains two exponential decay rates $\lambda_{1}$ and $\lambda_{2}$. The extrapolation of these rates to zero optical excitation allows the excited state radiative lifetime and shelving decay rates to be determined. The decay rate $\lambda_{2}$ as a function of optical power is shown in the inset of Fig. 5. The resulting values of $r_{31}^{0}$ and $r_{23}$ are 6.2 and $0.89 \mathrm{MHz}$, respectively, and are much smaller than the excited state decay rate, obtained from $\lambda_{1}, r_{21}^{0}=880 \mathrm{MHz}\left(\tau_{\text {rad }}=1.1 \mathrm{~ns}\right)$. We note that we have found several examples of the centers with similar ZPL which exhibit the same photophysical properties.

The single-photon emission count rate as a function of optical power $P_{\text {opt }}$ is shown for each emitter in Fig. 6. The measured count rates (corrected for the background) are given by the sum of the counts on the two APDs in the HBT setup. For the 744-, 756-, and 764-nm emitters, the saturation curves were fit according to Eq. (9) yielding saturation count rates $\phi_{\infty}$ of $2.1 \times 10^{6}, 3.2 \times 10^{6}$, and $1.3 \times 10^{6}$ counts $/ \mathrm{s}$, respectively. The saturation curve of the three-level 749-nm emitter was fit using Eq. (10), with the values of $r_{21}, r_{12}, r_{31}, r_{23}$ obtained from the fit to $g_{\text {meas }}^{(2)}(\tau)$ as a function of pump power and the value $\eta \eta_{\mathrm{QE}}$ left as fitting parameter.

Figure 7 shows a direct measurement of the lifetime recorded from the emitters using the pulsed excitation at $20-\mathrm{MHz}$ repetition rate. We note that the direct lifetime measurement characterizes the total decay rate from the excited state, which includes all the possible decay paths [19]. The single exponential fit to the fluorescence decay of each emitter, resulted in measured excited state total lifetimes of 4.1, 1.4, and 14.2 ns for the 744-, 749-, and 764-nm centers, respectively. Upon inspection of the fluorescence decay of

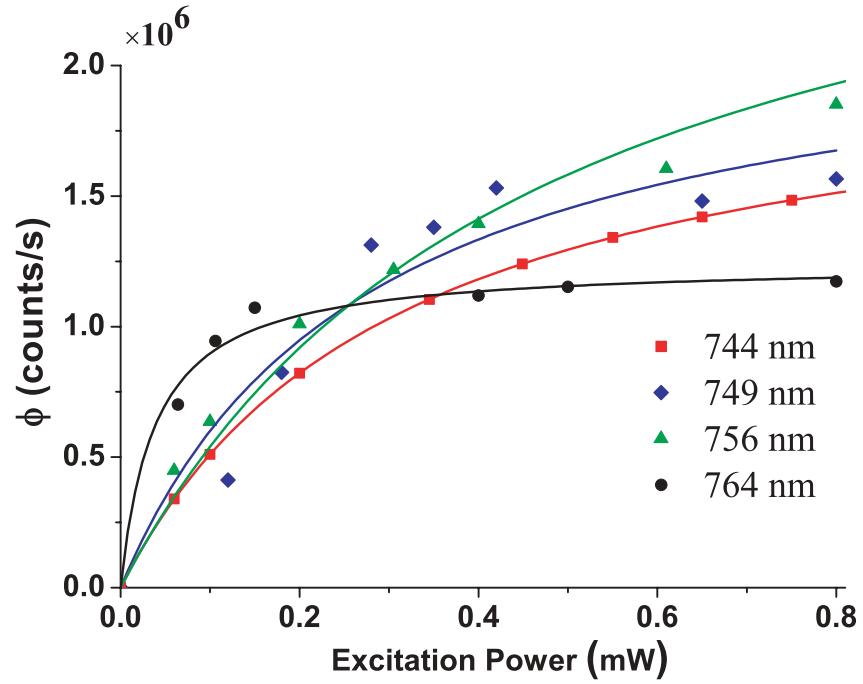

FIG. 6. (Color online) Measured saturation curves and fit according to Eq. (9) with estimated parameters of optical saturation power, $P_{\text {sat }}$, and single-photon count rate at saturation, $\phi_{\infty}$, for the centers at $744 \mathrm{~nm}$ (red squares) with $P_{\text {sat }}=311 \mu \mathrm{W}$ and $\phi_{\infty}=2.1 \times$ $10^{6}$ counts $/ \mathrm{s}$; at $756 \mathrm{~nm}$ (green triangles) with $P_{\text {sat }}=500 \mu \mathrm{W}$ and $\phi_{\infty}=3.2 \times 10^{5}$ counts $/ \mathrm{s}$; at $764 \mathrm{~nm}$ (black circles) with $P_{\text {sat }}=$ $40 \mu \mathrm{W}$ and $\phi_{\infty}=1.3 \times 10^{6}$ counts $/ \mathrm{s}$. For the center at $749 \mathrm{~nm}$ (blue diamonds) the saturation curve was fit by Eq. (10) with the estimated values $r_{21}, r_{12}, r_{31}, r_{23}$, and $\eta \eta_{\mathrm{QE}}$ used as fit parameter. From the fit a relative fluorescence quantum yield of $\eta_{\mathrm{QE}}^{3 \text { level }} / \eta_{\mathrm{QE}}^{\text {2level }}=0.24 \pm 0.08$ is obtained, assuming the collection efficiency constant.

the 744- and 749-nm centers there is a fast component of the fluorescence decay which occurs on a timescale less than $0.5 \mathrm{~ns}$, this component is attributed to the background within the emitting crystal. The lifetime of the emitter in this case is determined by fitting the fluorescence decay for times greater

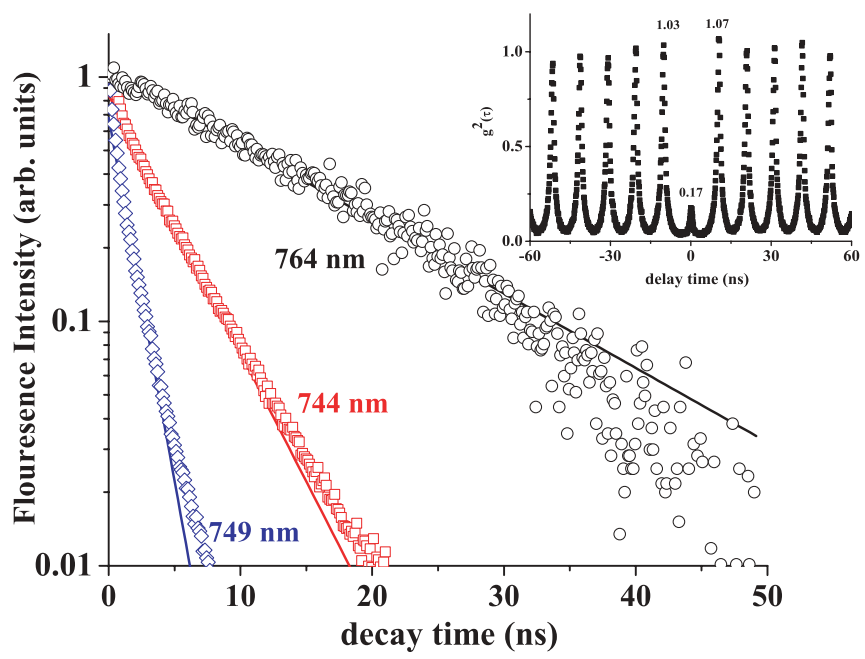

FIG. 7. (Color online) Direct measurement of the lifetime for the centers at $744 \mathrm{~nm}$ (red squares), $749 \mathrm{~nm}$ (blue diamonds), and $764 \mathrm{~nm}$ (black circles), using a pulsed laser at 20-MHz repetition rate with 200-ps pulse width. The data were fit with a single exponential. The deduced lifetimes of the centers are, respectively, $4.12 \pm 0.02 \mathrm{~ns}$, $1.41 \pm 0.02 \mathrm{~ns}$, and $14.2 \pm 0.2 \mathrm{~ns}$. (Inset) Antibunching measurement recorded from a single emitter at $749 \mathrm{~nm}$ under pulsed laser excitation at $40 \mathrm{MHz}$ and average power of $70 \mu \mathrm{W}$. 
TABLE I. Summary of the photophysical parameters (the $\tau_{\text {rad }}$ deduced from the cw corresponds to the radiative lifetime, while $\tau_{t}$ measured in the pulsed regime indicates the total lifetime).

\begin{tabular}{lcccccc}
\hline \hline System & $\lambda(\mathrm{nm})$ & $\phi_{\infty}($ counts $/ \mathrm{s})$ & $\tau_{t}(\mathrm{~ns})($ pulsed $)$ & $\tau_{\text {rad }}(\mathrm{ns})(\mathrm{cw})$ & $r_{31}(\mathrm{MHz})$ & $r_{23}(\mathrm{MHz})$ \\
\hline Two-level & 744 & $2.1 \times 10^{6}$ & $4.12 \pm 0.02$ & $3.8 \pm 0.3$ & & \\
Two-level & 756 & $3.2 \times 10^{6}$ & & $3.7 \pm 0.5$ & & \\
Two-level & 764 & $1.3 \times 10^{6}$ & $14.2 \pm 0.2$ & $13 \pm 1.5$ & & \\
Three-level & 749 & $2.7 \times 10^{6}$ & $1.41 \pm 0.02$ & $1.1 \pm 0.1$ & $6.2 \pm 0.6$ & $0.89 \pm 0.09$ \\
\hline \hline
\end{tabular}

than $0.5 \mathrm{~ns}$. The long fluorescence lifetime of the 764-nm center mitigates this effect and the decay can be described well by the single exponential fit. The measured lifetimes are in a good agreement with the lifetimes estimated from the cw measurements for the 744- and 749-nm emitters. The discrepancy with the 764-nm center can be due to the lack of the cw $g^{(2)}(\tau)$ measurements at excitation powers well below saturation, which affects the fit to a zero excitation power.

In the inset of Fig. 7 is an example of the second-order correlation function measurement under pulsed excitation from the 749-nm emitting crystal. The peak at $\tau=0$ is the probability of having more than one photon in the same pulse, showing single-photon emission $\left[g^{(2)}(0)=0.17\right]$. The observed deviation from zero is due to the background luminescence from the diamond nanocrystals, which is not negligible in this case. To reduce the unwanted background for practical applications, temporal filtering of our single-photon source can be implemented.

Table I summarizes the photophysical parameters for the emitters. It can be seen that the excited state lifetime, $\tau_{t}$, measured by direct measurement and the radiative lifetime deduced from the autocorrelation measurements are in agreement within our experimental uncertainty.

We turned our attention to the determination of the value of $\eta \eta_{\mathrm{QE}}$ for the two-level emitters and we compare this with the three-level emitter. In the case of a two-level system the term $\eta \eta_{\mathrm{QE}}$ can be measured directly, as each excitation pulse generates one emitted photon (without being trapped in the metastable state). In the condition where the laser excitation energy is above saturation, the temporal separation between laser pulses is longer than the typical detector dead time $(50 \mathrm{~ns})$ and the source lifetime $(14.2 \mathrm{~ns})$, the $\eta \eta_{\mathrm{QE}}$ can therefore be directly measured from the total count rate and the laser repetition rate $\eta \eta_{\mathrm{QE}}=\phi / R_{\text {rep }}$. As an example, exciting the $764 \mathrm{~nm}$ with a laser repetition rate of $10 \mathrm{MHz}, \eta \eta_{\mathrm{QE}}=0.015 \pm 0.001$ is obtained. Similarly, in the cw regime, $\eta \eta_{\mathrm{QE}}$ is given by $\eta \eta_{\mathrm{QE}}=\phi_{\infty} /\left(r_{21}^{0}\right)$. By calculating $\eta \eta_{\mathrm{QE}}$ for each two-level emitter, an average value from all the measurements $\eta \eta_{\mathrm{QE}}=0.013 \pm 0.004$ is obtained, in accord with the estimation from the pulsed measurements. The discrepancy in this value for various two-level emitters may be attributed mostly to a variation associated with the unknown dipole orientation of the emitter within the crystal and/or residual polarization-dependent background that can influence the total saturation count rate $\phi_{\infty}$. Since the emitters are embedded in nanocrystals, each absorption dipole has different orientation with respect to the excitation beam. Prior to each measurement, we optimized the excitation polarization to achieve maximum absorption cross section by rotating the half-wave plate controlling the incident beam polarization. The different orientation of the absorption dipole with respect to the incident beam was verified by the different optimal positions of the wave plate controlling the incident beam. Various emission dipoles were verified by the different optimal positions of the polarizer on the detection channel.

An indicative estimation of the collection efficiency of our setup can be obtained [10,11], considering in this case the subwavelength nanodiamond as a point source radiating at the air-glass interface. In our setup the total optical transmittance is $\approx 0.62$, given by the objective transmittance $(0.8)$, the filters F1 and F2 (0.97 and 0.98), the dichroic mirror (0.91), and the collimating lens (0.9). The detector efficiency is 0.65 and the multimode mode fiber coupling of 0.85 yielding a total detection efficiency of $\approx 0.55$. Finally considering an $\approx 0.2$ factor due to the spherical aberration of the objective (a nonimmersion oil objective is used) [11] and $\mathrm{a} \approx 0.2$ factor due to the optical surfaces (lens, filters, and dichroic), we estimate the collection efficiency results $\approx 0.014$.

We want to underline at this point that in order to establish an actual standard for fluorescence emission measurements, an absolute measurement of the fluorescence quantum yield in nanodiamonds, as reported for CdSe nanocrystals and single molecular dipole [28,29], would be required. In fact, even if there are circumstantial evidence of possible negligible nonradiative decay in $\mathrm{N}-V$ centers [30], this may not hold in any case. Moreover, an accurate measurement of the collection efficiency setup could be itself quite challenging. For the purpose of analyzing the efficiency of here presented emitters, we however determined the relative fluorescence quantum yield of the 749-nm emitter with respect to the two-level emitters. Assuming similar collection efficiency for all the emitters, we determined $\eta_{\mathrm{QE}}^{3-\text { level }} / \eta_{\mathrm{QE}}^{2 \text {-level }}=0.24 \pm 0.08$, nearly four times lower than the two-level emitters, as derived from the fit of the saturation curve with Eq. (9).

From our analysis, a two-level system with higher quantum efficiency demonstrates a clear advantage over a three-level system, for what concerns accurate measurement of fluorescence decay rates. In particular, the absence of an intersystem crossing rate could facilitate the accurate determination of the variation of radiative and nonradiative decay rates in the presence of modification from the bare nanocrystals induced by the environment.

The ratio between the average off and on periods of the source is given by the ratio $r_{23} / r_{31}$ which equals 0.14 for the 749-nm emitter. This value indicates that the probability of transition to the metastable state is moderate, as shown by the slight bunching of the $g^{(2)}(\tau)$ function [Fig. 4(b)]. Previous determinations associated with the NE8 complex in bulk natural diamond IIa [10,11] and CVD nanodiamond [12] 
showed $r_{23} / r_{31}$ of $2.8,1.6$, and 0.8 , respectively, justifying much lower saturation rates of NE8 with respect to the 749-nm center. In addition, the center possesses a shorter lifetime than NE8, which also contributes to the higher emission rates despite the moderately low fluorescence quantum yield.

The investigated two-level emitters possessed a ZPL with a FWHM of $\sim 10 \mathrm{~nm}$, while the three-level emitters exhibited a FWHM of $\sim 4 \mathrm{~nm}$.

The single emitting centers in the range $740-770 \mathrm{~nm}$ are believed to arise from chromium atoms within the diamond lattice. The charge state of the chromium complex and number of atoms involved is unknown. The explanation of what leads to two- or three-level behavior of a particular crystal is still under investigation. One may assume that the strain within the crystal may have a significant effect.

\section{CONCLUSION}

Optical characterization of the photophysics of optical centers attributed to chromium-related defects in submicron CVD-grown diamond crystals was performed. Additional two-level systems with ZPLs at 744 and $764 \mathrm{~nm}$ were identified and characterized. The fluorescence quantum yield of the three-level single emitter at $749 \mathrm{~nm}$ results four times lower with respect to the fluorescence quantum yield of the two-level emitters. The spectral width of the two-level emitters was $\sim 10 \mathrm{~nm}$, while the three-level emitters exhibited a FWHM of $\sim 4 \mathrm{~nm}$. This coupled with the short excited state lifetimes (1-14 ns) and the high brightness (up to $\mathrm{MHz}$ regime), brings these diamond centers much closer to a "practical single photon source on demand."

\section{ACKNOWLEDGMENTS}

The authors thank Mete Atature for helpful discussions. This work was supported by the Australian Research Council, the International Science Linkages Program of the Australian Department of Innovation, Industry, Science and Research (Project No. CG110039), and by the European Union Sixth Framework Program under the EQUIND IST-034368. A.D.G. received support from the Australian Research Council (Project No. DP0880466).
[1] S. Strauf, N. G. Stoltz, M. T. Rakher, L. A. Coldren, P. M. Petroff, and D. Bouwmeester, Nat. Photonics 1, 704 (2007).

[2] C. Santori, M. Pelton, G. Solomon, Y. Dale, and Y. Yamamoto, Phys. Rev. Lett. 86, 1502 (2001).

[3] C. Brunel, B. Lounis, P. Tamarat, and M. Orrit, Phys. Rev. Lett. 83, 2722 (1999).

[4] A. Tribu, G. Sallen, T. Aichele, R. Andre, J. P. Poizat, C. Bougerol, S. Tatarenko, and K. Kheng, Nano Lett. 8, 4326 (2008).

[5] C. Kurtsiefer, S. Mayer, P. Zarda, and H. Weinfurter, Phys. Rev. Lett. 85, 290 (2000).

[6] A. Beveratos, S. Kuhn, R. Brouri, T. Gacoin, J. P. Poizat, and P. Grangier, Eur. Phys. J. D 18, 191 (2002).

[7] A. M. Zaitsev, Optical Properties of Diamond: A Data Handbook (Springer, New York, 2001).

[8] F. Jelezko and J. Wrachtrup, Phys. Status Solidi 203, 3207 (2006).

[9] C. L. Wang, C. Kurtsiefer, H. Weinfurter, and B. Burchard, J. Phys. B 39, 37 (2006).

[10] T. Gaebel, I. Popa, A. Gruber, M. Domhan, F. Jelezko, and J. Wrachtrup, New J. Phys. 6, 98 (2004).

[11] E. Wu, V. Jacques, H. Zeng, P. Grangier, F. Treussard, and J. Roch, Opt. Express 14, 1296 (2006).

[12] E. Wu, J. Rabeau, G. Roger, F. Treussart, H. Zeng, P. Grangier, S. Prawer, and J. F. Roch, New J. Phys. 9, 434 (2007).

[13] I. Aharonovich, C. Zhou, A. Stacey, F. Treussart, J. F. Roch, and S. Prawer, Appl. Phys. Lett. 93, 243112 (2008).

[14] M. Wolfer, A. Kriele, O. A. Williams, H. Obloh, C.-C. Leancu, and C. E. Nebel, Phys. Status Solidi 206, 2012 (2009).

[15] I. Aharonovich, C. Zhou, A. Stacey, J. Orwa, S. Castelletto, D. Simpson, A. D. Greentree, F. Treussart, J.-F. Roch, and S. Prawer, Phys. Rev. B 79, 235316 (2009).

[16] D. A. Simpson, E. Ampem-Lassen, B. C. Gibson, S. Trpkovski, F. Hossain, S. T. Huntington, A. D. Greentree, L. C. L.
Hollenberg, and S. Prawer, Appl. Phys. Lett. 94, 203107 (2009).

[17] I. Aharonovich, S. Castelletto, D. A. Simpson, A. Stacey, J. McCallum, A. D. Greentree, and S. Prawer, Nano Lett. 9, 3191 (2009).

[18] J. Y. Cheung, C. J. Chunnilall, E. R. Woolliams, N. P. Fox, J. R. Mountford, J. Wang, and P. J. Thomas, J. Mod. Opt. 54, 373 (2007).

[19] S. Schietinger, M. Barth, T. Aichele, and O. Benson, Nano Lett. 9, 1694 (2009).

[20] C. H. Su, A. D. Greentree, and L. C. L. Hollenberg, Opt. Express 16, 6240 (2008).

[21] S. Schietinger, T. Aichele, and O. Benson, Nano Lett. 1, 3911 (2008).

[22] A. Greentree, B. A. Fairchild, F. Hossian, and S. Prawer, Materials Today 11, 22 (2008).

[23] W. Schmunk, H. Hofer, S. Peters, M. Rodenberger, and S. Kück, Radiometric Calibration of Single-Photon Counting Detectors, presented at the Single Photon Workshop 2009 conference, Boulder, Colorado, 2-6 Nov, 2009 (NIST, Boulder, 2009).

[24] S. C. Kitson, P. Jonsson, J. G. Rarity, and P. R. Tapster, Phys. Rev. A 58, 620 (1998).

[25] F. Treussart, A. Clouqueur, C. Grossman, and J. F. Roch, Opt. Lett. 26, 1504 (2001).

[26] A. Stacey, I. Aharonovich, S. Prawer, and J. E. Butler, Diam. Relat. Mater. 18, 51 (2009).

[27] A. M. Zaitsev, Phys. Rev. B 61, 12909 (2000).

[28] X. Brokmann, L. Coolen, M. Dahan, and J. P. Hermier, Phys. Rev. Lett. 93, 107403 (2004).

[29] B. C. Buchler, T. Kalkbrenner, C. Hettich, and V. Sandoghdar, Phys. Rev. Lett. 95, 063003 (2005).

[30] T. Gaebel, M. Domhan, C. Wittmann, I. Popa, F. Jelezko, J. Rabeau, A. Greentree, S. Prawer, E. Trajkov, P. Hemmer, and J. Wrachtrup, Appl. Phys. B: Lasers Opt. 82, 243 (2006). 